\title{
Bolt loose judging device for offshore wind turbine
}

\author{
Wang Fei*, Qu Jun \\ College of Energy and Mechanical Engineering, Shanghai University of Electric Power, Shanghai 200090, China
}

\begin{abstract}
For the problem of bolt loose in the long-term operation of offshore wind turbine, we proposed a bolt loose judging device for offshore wind turbine. The bolt loose judging device for offshore wind turbine included vibration sensor, signal receiving and transmitter, CCD sensor, data analyzer, power supply, the communication line and the power supply line. The combination of data analyzer and CCD sensor was used to analyze whether the prefabricated symbols on the bolts change, so as to judge whether the bolts are loose. The whole system (except vibration sensor) has been in standby and dormant state at ordinary times, which is more simple and reliable under the harsh conditions at sea.
\end{abstract}

\section{Introduction}

The operating environment of offshore wind turbine is worse than that of inland. In the normal operation of offshore wind turbine, and the bolts of the connecting parts are easy to loosen because of the long-term operation in the resultant force of various vibrations. When the bolts of main working parts are loose, it will cause severe shaking fault. The loose fixing bolts of the typical generator base will cause the wind turbine to vibrate violently. If the maintenance is not found in time, the steel wire rope cable can be pulled out and wind turbine will fall down and be damaged in case of serious accumulation over time ${ }^{[1-2]}$.

According to the existing literature ${ }^{[3-5]}$, the main causes of bolt loosening were as follows: (1) There is a deviation in the installation of main operating parts of the fan, which leads to a large vibration during the operation of the fan and the gradual loosening of the nuts of the bolts. (2) The bolts are not fastened in place, which leads to the gradual loosening of the nuts under the vibration. (3) The material characteristics of the bolts are not qualified, and the diameter of the bolts is gradually narrowed under the vibration, which leads to the gradual loosening of the nuts.

At present, the main method is regular inspection by the operation and maintenance personnel. Because the working environment of the wind turbine is at sea, compared with the onshore wind turbine, the transportation cost and labor cost are significantly increased. We proposed a bolt loosening judgment device for offshore wind turbine. The combination of data analyzer and CCD sensor is used to analyze whether the prefabricated symbols on the bolts change, so as to judge whether the bolts are loose, which has good economy. Compared with other methods of judging bolt looseness, the strategy of this method is simple, the whole system (except vibration sensor) has been in standby and dormant state at ordinary times, and the device is more simple and reliable under the harsh conditions at sea.

\section{Stress analysis of typical bolts}

Bolt connection was to clamp the connector by a large tightening pretension in the bolt rod, which was enough to produce a large friction force, so as to improve the integrity and rigidity of the connector. The typical highstrength bolt used for wind turbine tower was designed for shear resistance according to the friction type connection.

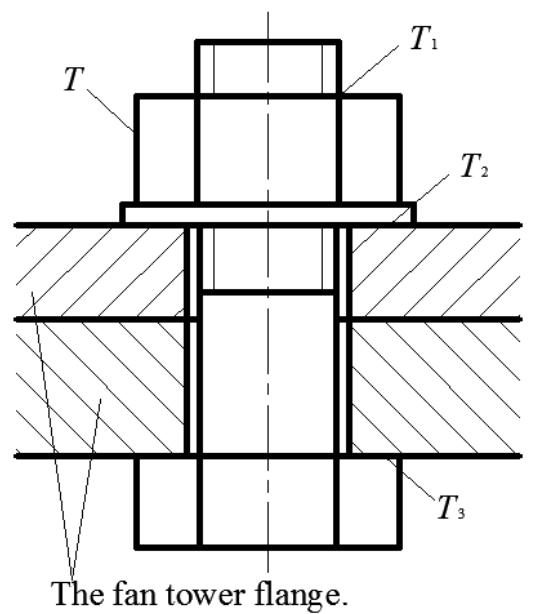

Fig. 1. The schematic stress analysis diagram of typical bolts.

The stress diagram of a typical wind turbine tower flange bolt was shown in Figure 1. Due to the existence

\footnotetext{
* Corresponding author: urrayu@163.com
} 
of tightening torque $T$, there is a preload $F_{0}$ between the bolt and wind turbine tower flange. The tightening torque $T$ is equal to the sum of the friction resistance moment $T_{1}$ between the screw pairs and the friction resistance moment $T_{2}$ between the ring end face of the nut and the support surface of wind turbine tower flange. The friction resistance moment $T_{3}$ between the annular end face of the bolt head and the support surface of the fan tower flange produces the reverse moment, which is equal to $T_{2}{ }^{[6]}$.

$$
\begin{aligned}
& T=T_{1}+T_{2} \\
& T_{2}=T_{3}
\end{aligned}
$$

And the expression of friction moment between screw pairs is

$$
T_{1}=F_{0} \frac{d_{2}}{2} \tan \left(\psi+\varphi_{V}\right)
$$

The symbol $d_{2}$ is pitch diameter of thread. The symbol $\psi$ is thread rising angle. The symbol $\varphi_{V}$ is equivalent friction angle of screw pair.

Also the friction moment between the nut and the flange surface of wind turbine tower is

$$
T_{2}=\frac{1}{3} \mu_{c} F_{0} \frac{D_{0}^{3}-d_{0}^{3}}{D_{0}^{2}-d_{0}^{2}}
$$

The symbol $\mu_{\mathrm{c}}$ is friction coefficient between nut and support surface. The symbol $D_{0}$ is outer diameter of bolt ring support surface. The symbol $d_{0}$ is bolt hole diameter.

Substituting formula (3) and formula (4) into formula (1), we can get:

$$
T=\frac{1}{2} F_{0}\left[d_{2} \tan \left(\psi+\varphi_{V}\right)+\frac{2}{3} \mu_{c} \frac{D_{0}^{3}-d_{0}^{3}}{D_{0}^{2}-d_{0}^{2}}\right]
$$

For a certain type of bolt, the parameter in the bracket of formula (5) is the fixed value, and it can be seen that the tightening torque $T$ is directly proportional to the pre tightening force $F$. Due to some reasons, the pre tightening force $F$ of the bolt decreases, which further reduces the tightening torque $T$ of the bolt, and finally the bolt loosens.

\section{The component of bolt loose judging device}

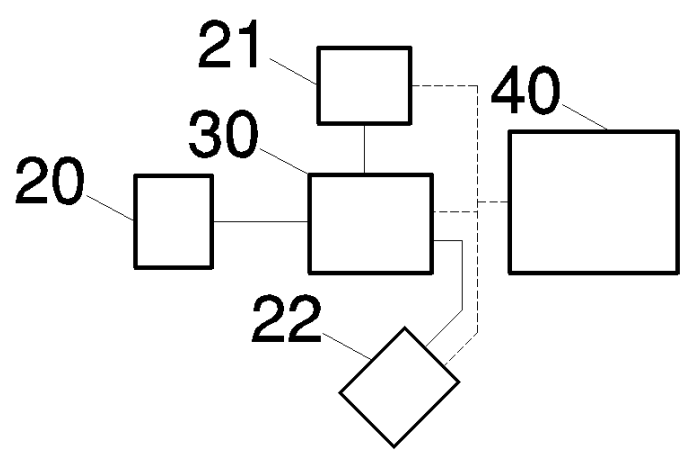

Fig. 2. Component sketch of bolt loose judging device. 20-vibration sensor, 21- signal receiving and transmitter, 22-CCD sensor, 30-data analyzer, 40-control device, 40power supply

As shown in Figure 2, bolt loose judging device for offshore wind turbine included vibration sensor, signal receiving and transmitter, $\mathrm{CCD}$ sensor, data analyzer, power supply, the communication line and the power supply line.

Data analyzer was connected with vibration sensor, signal receiving and transmitter and CCD sensor respectively through the communication line. Power supply was connected with signal receiving and transmitter, data analyzer and CCD sensor respectively through the power supply line.

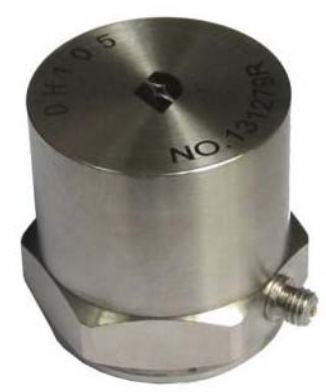

Fig. 3. A typical piezoelectric acceleration sensor.

The methods of vibration measurement in engineering include mechanical method, optical method and electrical method. Among them, the most widely used electrical measurement method was to convert mechanical vibration into electricity, and then measure and convert the electricity to make it a signal output. According to their working principles, the specific vibration measuring sensors can be divided into piezoelectric, piezoresistive, capacitive, inductive and photoelectric types. As shown in Figure 3, Piezoelectric acceleration sensor was the most commonly used vibration measurement sensor because of its wide measuring frequency range, large measuring range, small volume, light weight, small impact on the measured parts and convenient installation and use. We choosed piezoelectric acceleration sensor here.

\section{Operation method of the device}

\subsection{Preliminary commissioning preparation}




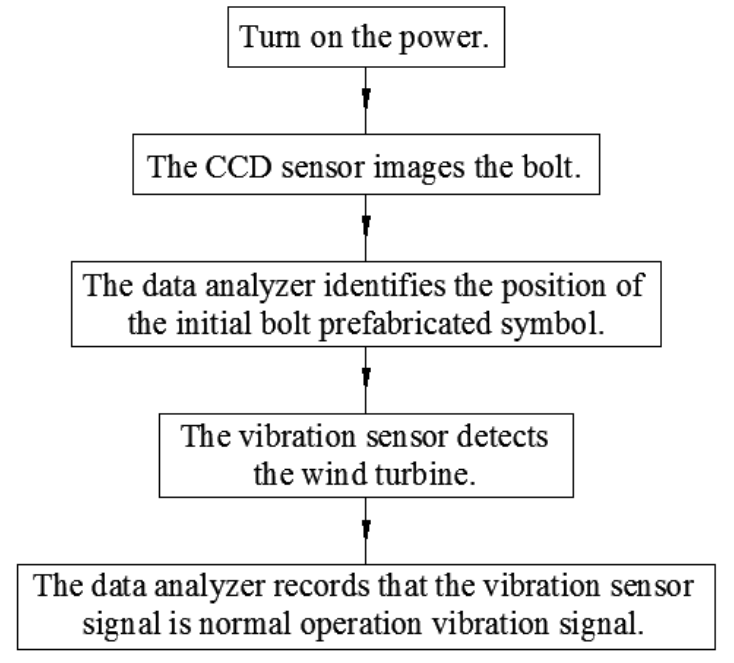

Fig. 4. Flow chart of preliminary commissioning preparation.

As shown in Figure 4, the power supply was turned on first, and the vibration sensor, signal receiving and transmitter, CCD sensor and data analyzer are in working state. The CCD sensor imaged the bolt, which transmits the initial state image of the bolt to the data analyzer through the communication line. As shown in Figure 5, the data analyzer identified the position of the initial bolt prefabricated symbol in the initial state image of the bolt, and the CCD sensor entered the standby sleep state.

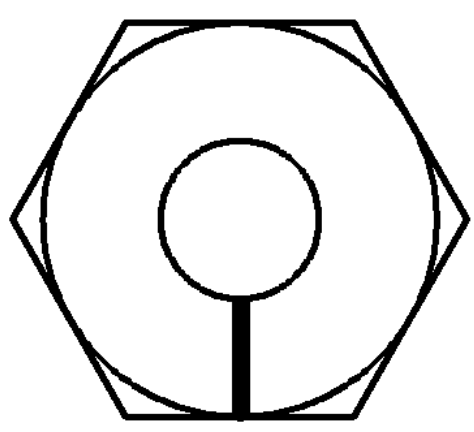

Fig. 5. The position of the initial bolt prefabricated symbol in the initial state image of the bolt.

During the normal operation of the wind turbine, the vibration sensor detected the wind turbine in real time and transmits the vibration signal to the data analyzer through the communication line. The data analyzer recorded that the vibration sensor signal is normal operation vibration signal at this time.

\subsection{Daily maintenance and inspection state}

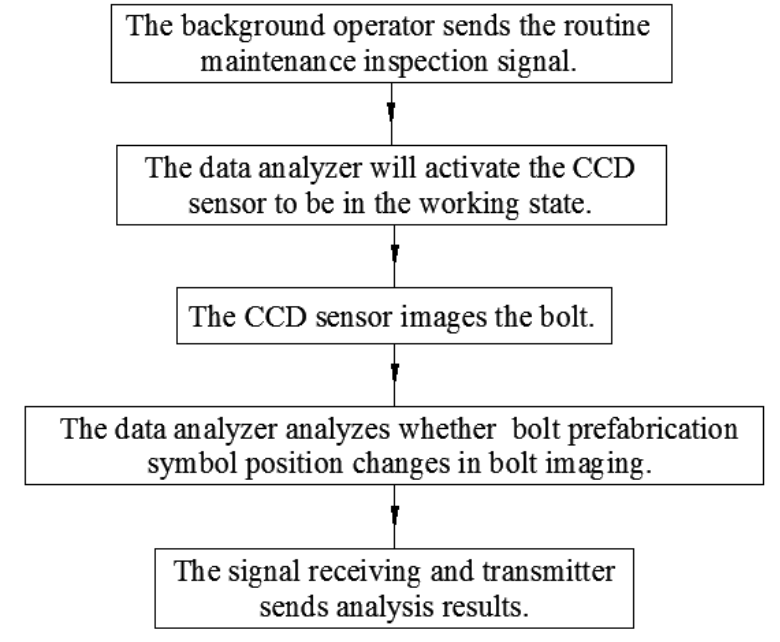

Fig. 6. Flow chart of daily maintenance and inspection state.

As shown in Figure 6, at the end of the routine maintenance inspection cycle, the background operator sended the routine maintenance inspection signal to the signal receiving and transmitter. The signal receiving and transmitter will transmit the daily maintenance and inspection signal to the data analyzer through the communication line, and then the data analyzer will activate the CCD sensor to be in the working state through the communication line. The CCD sensor will image the bolt state through the communication line to the data analyzer, and the CCD sensor will enter the standby sleep state.

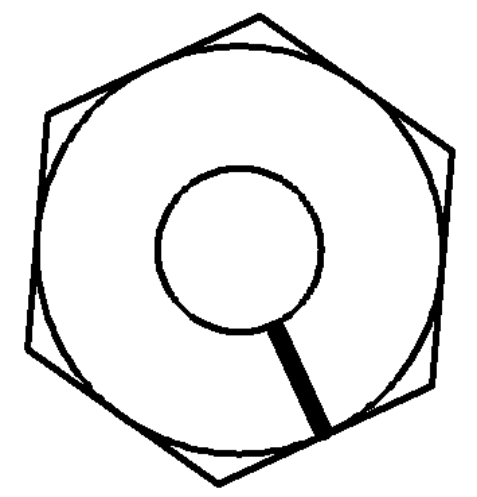

Fig. 7. The bolt prefabricated symbol position A in bolt status imaging.

As shown in Figure 7, the data analyzer identified bolt prefabricated symbol position $\mathrm{A}$ in bolt status imaging, which was different from the initial bolt prefabricated symbol position. The data analyzer connected the signal receiving and transmitter through the communication line, and the signal receiving and transmitter transmitted the signal of wind turbine bolt loosening. When the data analyzer recognizes that the position of bolt prefabrication symbol is the same as that of the initial bolt prefabrication symbol, the signal receiving and transmitter will transmit the normal signal of wind turbine bolt. 


\subsection{Severe shaking state of wind turbine}

As shown in Figure 8, when the wind turbine shakes violently due to loose bolts, the vibration sensor will transmit the vibration signal $\mathrm{B}$ of the wind turbine to the data analyzer through the communication line. The data analyzer compared the vibration signal B of wind turbine operation with that of normal operation, and concluded that the vibration signal B of wind turbine operation is the vibration signal of abnormal operation.

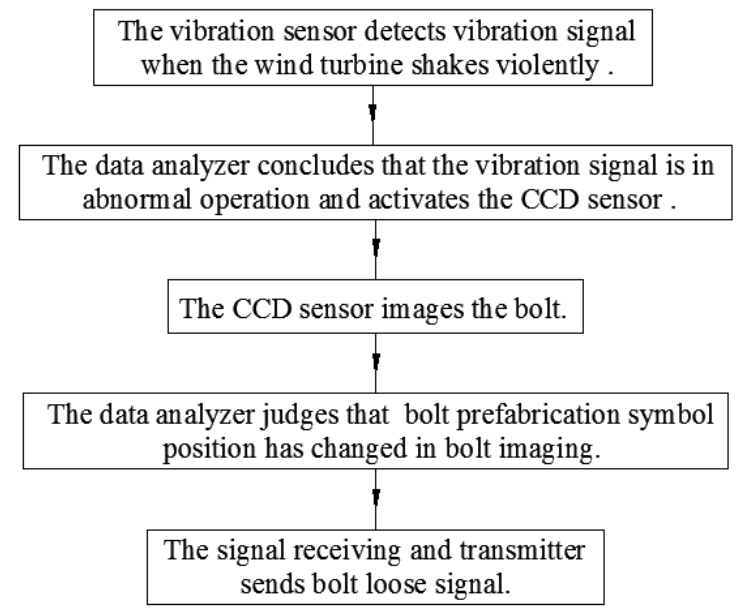

Fig. 8. Flow chart of severe shaking state of wind turbine.

The data analyzer activated the CCD sensor to be in the working state through the communication line, and the CCD sensor transmitted the image of the bolt state to the data analyzer through the communication line. The CCD sensor then entered the standby sleep state. As shown in Figure 8, the data analyzer identified the position $\mathrm{B}$ of the bolt prefabrication symbol in the image of bolt status, which was different from the position of the initial bolt prefabrication symbol. The data analyzer connected the signal receiving and transmitter through the communication line, and the signal receiving and transmitter transmitted the signal of wind turbine bolt loosening.

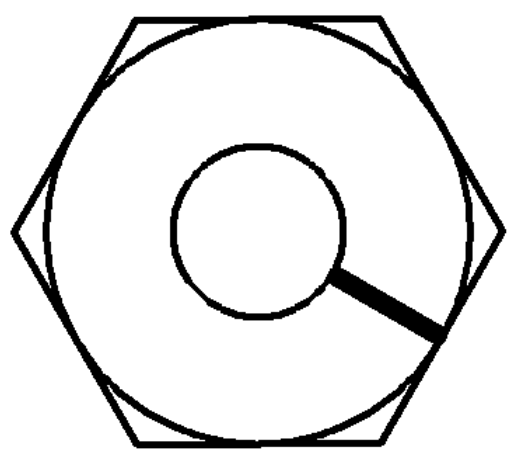

Fig. 9. The bolt prefabricated symbol position B in bolt status imaging.

\section{Conclusions}

The bolt loose judging device for offshore wind turbine included vibration sensor, signal receiving and transmitter, CCD sensor, data analyzer, power supply, the communication line and the power supply line. The device included daily maintenance and inspection state and severe shaking state of wind turbine. The combination of data analyzer and CCD sensor was used to analyze whether the prefabricated symbols on the bolts change, so as to judge whether the bolts are loose. The whole system (except vibration sensor) has been in standby and dormant state at ordinary times, which is more simple and reliable under the harsh conditions at sea.

\section{References}

1. J.M. Liao, Z. Zhou, C. Zhu, Z.L. Shang, J. Lu, CHN. Mach. Elec. 37, 5 (2019)

2. Y. Zhao, B. Han, G. L. Fang, CHN. Thermal Power Generation. 45, 10 (2016)

3. B.Y. Hou, Q.H. Qiu, B. Wang, CHN. J. Vib. Shock. 34, 23 (2015)

4. Y.G. Li, X.N. Sun, Y.Y. Gao, CHN. J. Vib. Shock. 34, 1 (2015)

5. T. Wei, K. Wu, J.W. Huang, CHN. Mach. Elec.8 (2013)

6. L.G. Pu, M.G. Ji, Mechanical design version 7 (HEP, Beijing, 2001) 